\title{
Numerical Modeling Approach of an Air-launched AUV Initially Impacting on Water
}

\author{
WANG Yonghu \\ Civil Aviation Flight University of China \\ Guanghan Sichuan, China \\ wangyh.cn@gmail.com
}

\begin{abstract}
Water-entry impact of air-launched AUV (AL-AUV) is considered as a time-depended strongly nonlinear fluidstructure Interaction phenomenon. Numerical modeling technique of AL-AUV on its initial water-entry impact is given by using the Multi-Material ALE-Lagrange coupling algorithm. The key aim is focused on the numerical modeling approach taken in detail to dealing with water-entry impact. Based upon this, water-entry impact loads and effect of fluid on the impact responses are also specially discussed. Numerical results show that the exact dynamic characteristics can be obtained in effect by modeling the appropriate waterentry numerical model and the right resolve method. It is helpful to the structure design and to analyze the later hydroballistics.
\end{abstract}

Keywords- AL-AUV; Water-entry impact; ANSYS/LS-DYNA; Coupling algorithm; Numerical modeling

\section{INTRODUCTION}

AL-AUV water-entry impacting problem is of great concern and belongs to the fluid-structure interaction (FSI) research area such as ship slamming, aircraft ditching, recovery module and lifeboat water impacting, etc. It is common to divide the phenomenon of water entry into the shock wave phase, the flow forming phase, the closed cavity phase and the collapsing cavity phase. The fist two phases is great emphasis placed on numerical analysis because the main body and the inner electronic instruments will be destroyed or fail by the huge impacting accelerations [1].

The majority of researchers have focused on water impacting through analytical, experimental, and finite element methods, etc. Th. von Karman and H. Wagner were the pioneers historically in studying the vertical water impact problems and estimated the impact acceleration base on the conservation of momentum [2]. The large following make extensions to get a solution by analytical, experimental and numerical methods and some of the methods are widely applied in 2D and 3D solutions [3]. But analytical results including fluid and structure deformation are not available if 3D geometries impacting on water. Lots of numerical methods have been adapted to the impact on water since the seventies of last century [4]. The main merits of numerical simulation are able to deal with problem of fluid-structure interaction and provide valuable insight into some physical phenomenon such as cavitations and jet flow. A set of airdropped AUV, REMUS designed by WHOI (Woods Hole

This Research is Supported by CAFUC Normal Foundation Project (J2011-03).
Oceanographic Institution) has been constructed to numerically estimate the hydrodynamic impact loads [5].

In this paper, the emphasis was put on the explicit nonlinear dynamic finite element code-ANSYS/LS-DYNA to numerically simulate hydrodynamics characteristics of AL-AUV on its water-entry impact. The numerical method to predict the responses due to the water-impact is addressed and verified to be useful. The impact accelerations and fluid formulation predicted efficiently are important for design of AL-AUV and other impactors.

\section{NUMERICAL MODELING}

The finite element analysis (FEA) method has been the revolution of numerical simulation for FSI problem. For the core competency of LS-DYNA lies in highly nonlinear transient dynamic FEA using explicit time integration [6], it is the most popular time-depended explicit solver in LSDYNA package for the AL-AUV water impacting simulation in this research. The explicit nonlinear dynamic finite element code has ability to capture the physics of short-duration events for structures that undergo highly nonlinear, transient dynamic forces. With ANSYS/LSDYNA and other tools, we can gain insight into how a structure responds when subjected to severe loads.

\section{A. Description and Hypothesis}

AL-AUV is designed based on the traditional torpedo shaped AUV, REMUS (Remote Environmental Monitoring Units), which is deployed from a helicopter, resulting in free-fall impacting on water. The water impacting hydrodynamic problem is depicted as shown in Fig. 1, a 3D air-dropped AUV penetrates the still water with water-entry angle , angle of attack , and initial speed which is parallel to the rotation axis of AUV.

The body-fixed right-hand Cartesian coordinate system $O-X Y Z$ is used with $Y$ located on $C G$ and points vertically upward. The axis $X$ is along the initial undisturbed free surface. The fluid problem is considered an idea fluid (incompressible, inviscid and irrotational), whereas AL-AUV is rigid body. The system of unit is adopted by $c m-g-\mu \mathrm{s}$, in which time is $\mu \mathrm{s}$, displacement is and accelerated speed is $\mathrm{cm} / \mu \mathrm{s}^{2}$. 


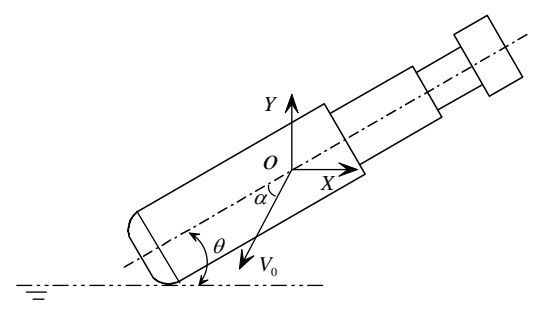

Fig. 1 Sketch of the water-entry attitude of AL-AUV

\section{B. Fluid-Structure Interaction Algorithm}

The template is used to format your paper and style the text. All margins, column widths, line spaces, and text fonts are prescribed; please do not alter them. You may note peculiarities. For example, the head margin in this template measures proportionately more than is customary. This measurement and others are deliberate, using specifications that anticipate your paper as one part of the entire proceedings, and not as an independent document. Please do not revise any of the current designations.

The selection of fluid-structure interaction algorithm is made based on the relationship between the computation efficiency and the precision of solution. The Lagrange approach is the most commonly used computationally and the fastest to represent structures. But it is not appropriate for modeling fluid or fluid-like behavior owing to the very large deformation of the material because mesh distortion causes smaller time step and numerical instability. The material is fixed to the mesh and moves with the grid, while the Euler approach is the material moves the fixed mesh. The Euler method is ideal for modeling severe deformations of fluid such as liquids and gases.

The physical process involving fluids or fluid-like behavior may always be modeled in more than ways. Arbitrary Lagrange-Euler (ALE) combines Lagrange and Euler approach, which involves a combination of the advantages of Lagrange and Euler while eliminating some of their shortcomings. It is much more suited to modeling of fluid or fluid-like behavior such as modeling the fluid domain and surrounding void in the paper. In LS-DYNA, Lagrange and Euler/ALE solution methods can be combined in the same model and FSI may be handled by a coupling algorithm. Lagrange, Euler and ALE are combined in a single problem to produce the most efficient and highest accuracy results as possible because the ALE mesh moves exactly without the material and the advection of material per time step is less.

So in these codes, the AL-AUV is practically modeled using the classical Lagrange algorithm in the crash simulation, whereas the fluid medium is modeled using the Euler/ALE algorithm, i.e. Multi-Material ALE method. A 3step of computational cycle procedure adopted by coupling algorithm is as follows.

Step 1: The first part of Lagrange time step is taken, causing the mesh distortion accompanied with the fluid flow. It is satisfied with the demand of the boundary conditions after the fluid deformation. Re-meshing of the internal elements is run in order to maintain the topology relationship of the meshes.

Step 2: Advection Step is drawn support to help the grid state variables (density, energy, stress tensor, etc.) of the deformed material configuration and the contact velocity vector to be transported onto the re-meshing grids. The coupling between the Lagrange mesh and ALE mesh serves to generate several of parameters such as the coupling forces which LS-DYNA solver automatically computes based on a penalty method.

Step 3: The last step is the implementation of ALE calculations in every time step. Coupling surface will results in motion deformation together with the common boundary between Lagrange elements and the Euler elements, which the Euler elements can move in a wide range. Elements are allowed to move and distort, resulting in the minimum advection. This minimizes energy dissipation and speeds up run time.

In the end, the quality of the results and the runtime of computation depend on the performance of the coupling algorithm utilized. And attention is paid to the problem of numerical stability and mesh deformation in the framework of the ALE technique.

\section{Numerical Modeling Technique}

The LS-DYNA explicit finite element program and the powerful pre- and post-processing capabilities are integrated into ANSYS/LS-DYNA, which is used to model the structure and fluid in ANSYS. We obtain the solution via LS-DYNA, and review results using the LS-PREPOST as the post-processing tool.

AL-AUV structure is modeled only thought the configuration directly impacting on water, regardless of the inner instruments. AL-AUV is defined as a rigid body using the EDMP command. The most merit is the rigid bodies used in the finite element model can greatly reduce the computation time required to perform the explicit analysis.

The degrees of freedom of the rigid body only have six degrees of freedom because all the nodes in the body are concentrated on the center of weight. At every time step, all the nodal forces and moments are summed to the forces and moments of the rigid body. And the same time, *PART_INERTIA card is used to define the center of gravity, mass, initial velocity in the global system of coordinates and the moment of inertia.

It is recommended that AUV modeled by a classical Lagrange technique in order to relieve of the timeconsuming, using shell elements and fluid (that is, air and water) is modeled by the ALE technique, using 8-node hexahedral solid elements. The finite element model of ALAUV is shown in Fig. 2. AL-AUV is discretized with up to 3266 4-node Lagrange shell elements. The air and water are discretized with hexahedral solid164 elements which amount depend on the different computational cases. 
Air on top of the water must be modeled by ALE mesh to allow the water to form the jet flow that occurs in an impacting. MAT_NULL card is used to define water behavior. Water and air are referred as Multi-Material to be modeled using the ALE formulation (ELFORM=11 in *SECTION_SOLID). *SECTION_SOILD_ALE here is defined the properties of elements to mark algorithms.

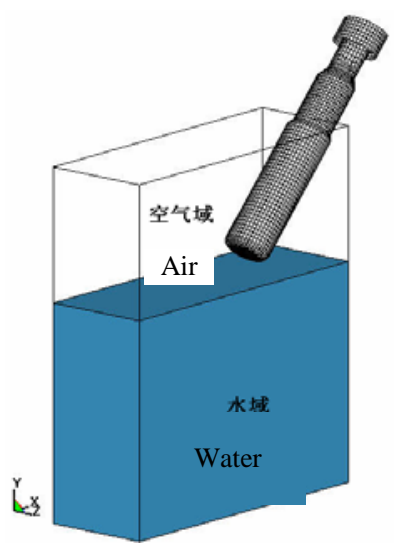

Fig. 2 3D numerical model of the full-scale AL-AUV and the fluid block

In numerical simulation, the coupling is done by the card CONSTRAINED_LAGRANGE_IN SOLID whose algorithm is ALE coupling algorithm. The Lagrange mesh does not share nodes with ALE interface surface.

There is the compatibility between mesh grid sizes used to model the fluid and the structure model. The Lagrange mesh is finer than Euler mesh to prevent leakage in the coupling [6]. The influence of the relation between solid element size and shell element size is important to gain more ability and more precise solution. So it is necessary that the ratio of element types available must be determined before appropriately building numerical models.

Thinking about high water-entry velocity of AUV, the dimension of air and water domain respectively is determined by the penetration depth and the jet flow subjected to water-entry impact. The penetration depth can be recommended to define with the time step using the command *CONTROL_TIMESTEP. Nonreflecting boundaries are available to represent the infinite fluid domain in order to limit the overall size of the fluid model and prevent stress wave reflections from the influence on the numerical results.

In the paper, the numerical computational flow chart is shown Fig. 3. At first Lagrange element sizes are given based on the structure dimension. And then some sets of size ratios impacted on the solutions are analyzed through changing the mesh size of fluid. At last, an optimal size ratio is selected for later simulation.

The numerical results can be reviewed by the two ANSYS postprocessors, POST1 and POST26, but here LSPREPOST is used for a postprocessor with LS-DYNA. The user interface is designed to be both efficient and intuitive. Log files generated by ANSYS preprocessor are operated to output the $\mathrm{K}$ files. The $\mathrm{K}$ files cannot directly be operated by the LS_DYNA solver because some keywords and parameters in the $\mathrm{K}$ files should be edited by hand.

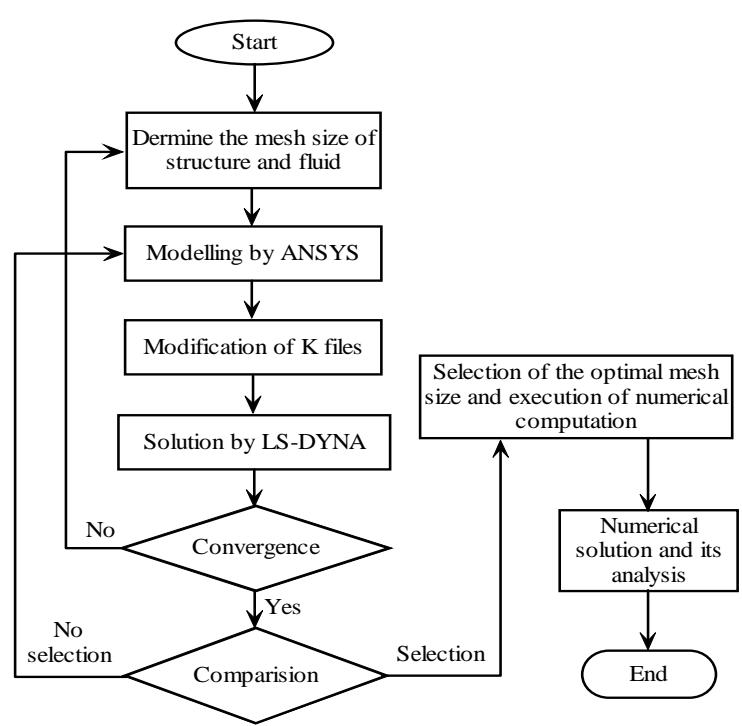

Fig. 3 Flow chart of numerical computation for AL-AUV water impacting

To summarize, the whole process of numerical simulation of water-entry impact is given as follows. The first is the creation of AUV and fluid models, meshing, boundary condition and initial conditions, and creating PART, K files output and edition are done. Second, new K files are given by GUI or DOS to LS-DYNA solver for deep computation. Third, LS-PREPOST handles the numerical results and displays the images or movies.

\section{NUMERICAL SOLUTION AND ANALYSIS}

In order to ensure the accuracy and effectiveness of the numerical simulation, the dimensions of solid domain and meshing method are unchanging in each simulation case. Fluid domain size, including air and water, is determined based on the horizontal displacement predicted in advanced based on water-entry velocity and computation time. The different sizes of fluid domain mean the different amounts of mesh because of the same mesh size. The technique is good for displaying all the jet flow in the air domain.

The initial conditions are listed in TABLE I, including four simulation cases, i.e. case A, case B, case C and case D. The initial parameters are water-entry angles and water-entry velocities and so on. The water impacting accelerations and time of accelerations are numerically computed by ALE coupling algorithm mentioned. According to results in the table, it can be shown that the influences of the water-entry angles on the impacting accelerations and the peak time of accelerations with the same water-entry velocities are significant. 
TABLE I. LIST OF NUMERICAL RESULTS FROM VARIOUS CASES

\begin{tabular}{|c|c|c|c|c|}
\hline \multirow{7}{*}{ Parameters } & $\begin{array}{c}\text { Water } \\
\text { entry } \\
\text { angle } \\
\left.\theta \mathbf{(}^{\circ}\right)\end{array}$ & $\begin{array}{c}\text { Water entry } \\
\text { velocity } \\
V_{0} \mathbf{( m / s )}\end{array}$ & $\begin{array}{c}\text { Maximum } \\
\text { acceleration } \\
\left(\mathbf{1 0 - 7} \mathbf{c m} / \mathbf{s}^{\mathbf{2}}\right)\end{array}$ & $\begin{array}{c}\text { Time of } \\
\text { maximum } \\
\text { acceleration } \\
\mathbf{( s )}\end{array}$ \\
\hline \multirow{2}{*}{$\begin{array}{c}\text { Numerical } \\
\text { results }\end{array}$} & 50 & 150 & 5.6 & 802 \\
\cline { 2 - 5 } & 20 & 150 & 4.1 & 1500 \\
\cline { 2 - 5 } & 12 & 160 & 3.6 & 2300 \\
\hline
\end{tabular}

The impact scenario of water propagation and load time history curve at the initial stage are gained with ANSYS/LSDYNA as shown in Fig. 4 and Fig. 5.

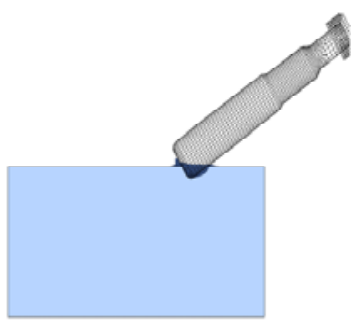

(1)

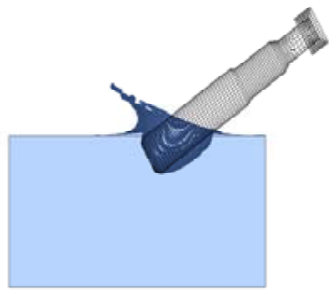

(3)

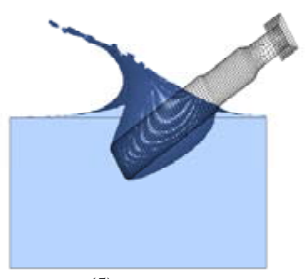

(5)

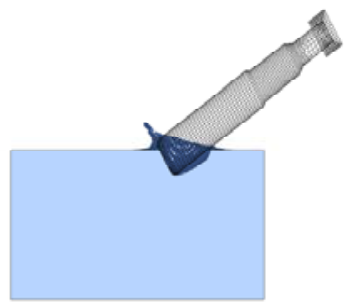

(2)

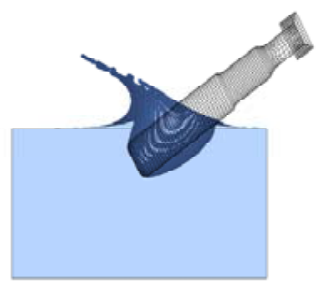

(4)

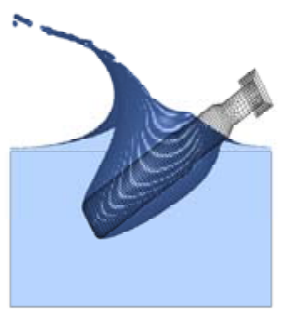

(6)
Fig. 4 Water propagation of AL-AUV penetrating water at initial water-entry angle $30^{\circ}$

The head configuration, the special disk nosed head, will greatly influence the hydrodynamics of water entry. It is reasonable that it exits the cavitations when impacting on water. It is very difficulty to be solved by some analytical methods, but it can be simulated by computer aided technology such as LS-DYNA program codes. The jet flow and cavitations of oblique water-entry of AL-AUV are shown in Fig. 4, which shows six frames depicting AL-AUV impacting the water at water-entry angle of 30 and $150 \mathrm{~m} / \mathrm{s}$.
From the series of figures, we also can see that the splash effects can be numerically reproduced very well by the adoption of the coupling algorithm. The 3D scenario of cavitations is particularly visualized to display its creation, its growing and its propagation. The collaboration of cavity wall and the shell of AL-AUV actuate a bend torque, which change the attitude of water entry. It takes responsibility for the emergence of whip and ricochet behaviors. And it should be avoided if the phenomenon becomes serious.

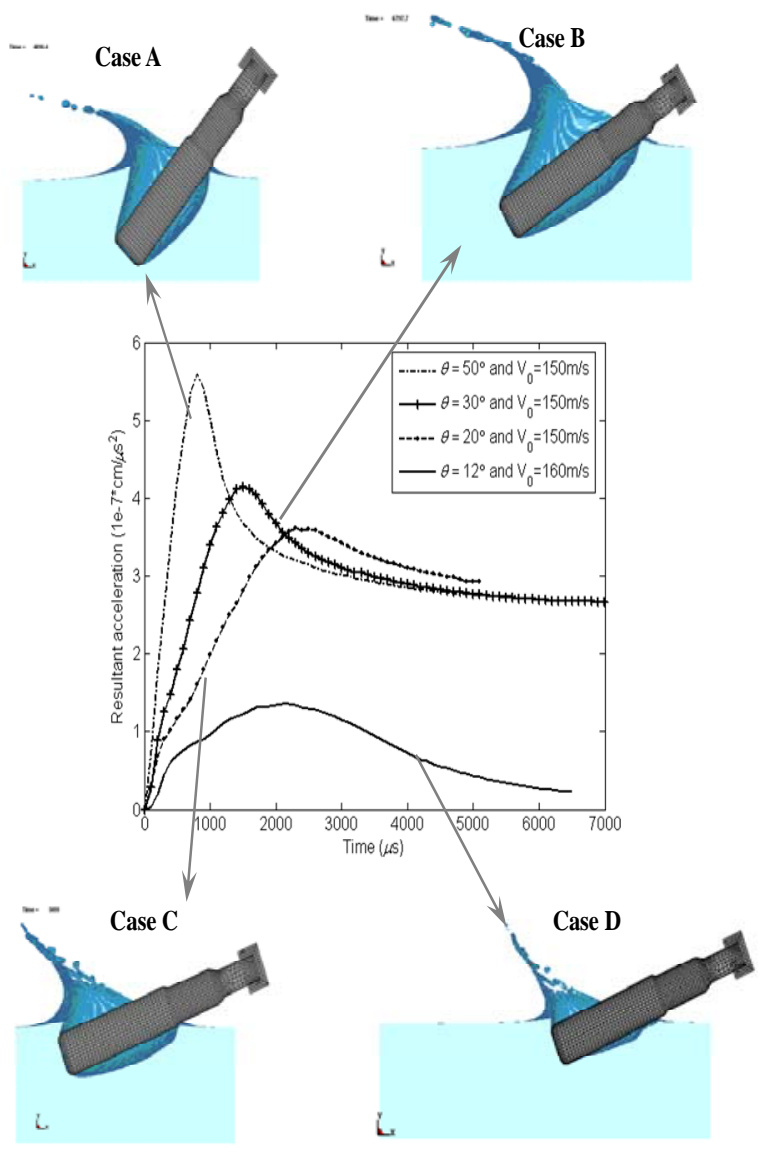

Fig. 5 Influence of various water-entry angles and velocities on the accelerations time histories

The four acceleration time history curves are shown in Fig. 5. It can be seen that acceleration and load peak value increase correspondingly as the water-entry angle increases, and the peak position for the small entry angle appears correspondingly a little later. Water-entry angle, initial velocity and attitude have respective influences on the calculated curves. The water-entry impacting time belongs to the level of a millisecond. It is obvious that the vertical water-entry impacting will cause the biggest peak value, which is understandable. The acceleration plateau following the peak is presented in each simulation cases. 


\section{CONCLUSION}

AUV is un-tethered moving platforms used widely and helpfully for survey operations and so on. This paper thoroughly presents the application of an alternative approach in ANSYS/LS-DYNA to numerically simulate the water-entry impacting problem of AL-AUV. Numerical analyses are for computing the acceleration and display 3D moving jet flow based on the Multi-Material ALE-Lagrange coupling method. The coupling algorithm well suited to this type of simulation because the large deformations it faced are difficult to be solved by some analytical methods.

Experiments on the four numerical cases are preformed based on the modeling step depicted and details above. The results illustrate that the coupling algorithm is a good approach to utilize when an AL-AUV initially impacts on water. And it reveals that the exact dynamic characteristics can be obtained by modeling the appropriate water-entry numerical model and the right resolve method. The hydrodynamic characteristic depends on the configuration of the impactor and the initial conditions of the impact, especially like water-entry velocity, angle, etc. It has a great influence on the ballistic behavior and the structure design.

The recommendations on the future direction of the technique for this study are made that various parametric studies should be conducted to investigate the effect of the simulation parameters on the result obtained.

\section{ACKNOWLEDGEMENT}

The authors greatly appreciate the strong support for the research from the Marine College, NWPU because of the offer of the finite element code-ANSYS/LS-DYNA.

\section{REFERENCES}

[1] Yonghu Wang, Xiuhua Shi, et al, "Modeling and cushion analysis of oblique water entry with high velocity.” Mechanical Science and Technology for Aerospace Engineering, vol. 27, pp. 766-769, 2008 (in Chinese).

[2] Man-Sung Park, Young-Rae Jung, and Warn-Gyn Park, "Numerical study of impact force and ricochet behavior of high speed water-entry bodies," Computers\&Fluids, vol. 32, pp. 939-951, 2003.

[3] J. G. Waugh, "Water-entry pitch modeling. Journal of Hydronautics," vol. 2, pp. 87-92, 1968.

[4] Zhaoyu Wei, Xiuhua Shi, et al, "The oblique water entry impact of a torpedo and its ballistic trajectory simulation,” International Journal of Numerical Analysis and Modeling, vol. 9, pp. 312-325, 2012.

[5] Stephen Michael Roe, "Numerical and experimental analysis of initial water impact of an air-dropped REMUS AUV," MIT MS thesis, 2005.

[6] J. O. Hallquist, "LS-DYNA user's manual v. 970,” Livermore Software Technology Company, 2004. 